\title{
An Alternative Theory on the Spacetime of Non-inertial Reference Frame
}

\author{
Gordon $\mathrm{Liu}^{1}$ \\ ${ }^{1}$ Copernicus Institute for Physics and Astronomy, Melbourne, Australia \\ Correspondence: Gordon Liu, Copernicus Institute for Physics and Astronomy, Melbourne, Australia. 38 Carolyn \\ Cres, Bundoora, VIC 3083, Australia. Tel: 61-431143618. E-mail: gordonliu168@gmail.com
}

Received: June 22, 2017

Accepted: August 29, $2017 \quad$ Online Published: September 11, 2017

doi:10.5539/apr.v9n5p90

URL: https://doi.org/10.5539/apr.v9n5p90

\begin{abstract}
In present paper, we have proposed an alternative theory on the spacetime of non-inertial reference frame (NRF) which bases on the requirement of general completeness (RGC) and the principle of equality of all reference frames (PERF). The RGC is that the physical equations used to describe the dynamics of matter and/or fields should include the descriptions that not only the matter and/or fields are at rest, but also they move relative to this reference frame, and the structure of the spacetime of reference frame has been considered. The PERF is that any reference frame can be used to describe the motion of matter and/or fields. The spacetime of NRF is inhomogeneous and deformed caused by the accelerating motion of the reference frame. The inertial force is the manifestation of deformed spacetime. The Riemann curvature tensor of the spacetime of NRF equals zero, but the RiemannChristoffel symbol never vanishs no matter what coordinate system is selected in the NRF. The physical equations satisfied the RGC remain covariance under the coordinate transformation between the reference frames. Mach's principle is incorrect. The problem of spacetime of NRF can be solved without considering gravitation.
\end{abstract}

Keywords: Relativity, Non-inertial Reference Frame, Spacetime, Riemann Spacetime, Gravitation, Mach Principle

\section{Introduction}

After establishing the theory of the spacetime of inertial reference frames (IRF), Special Relativity (SR), Einstein faced two problems: 1) how to extend SR to non-inertial reference frame (NRF); 2) Newtonian law of gravity did not satisfy Lorentz covariance. The problems headed Einstein toward to propose the principle of equivalence and the principle of general relativity, thereby, to develop General Relativity (GR). Einstein tried to solve the two problems by proposing GR and mixed the problem of NRF with gravitation. People often think that this is Einstein's masterpiece, killing two birds with one stone. Now the most physicists hold following Einstein's opinions: 1) the inertial force can be regarded as a kind of gravitation (the principle of equivalence); 2) a NRF can be considered as an IRF with a gravitational field (the premise of the principle of general relativity); 3) the source of the inertial force is faraway galaxies (Mach principle) (Einstein, 1920, 1970; Feynman, 1995; Liu, 2004, pp.8; Misner, Thorne, \& Wheeler, 1973; Moller, 1952; Weinberg, 1972). Some authors even tried to obtain the spacetime metric of the NRF by solving the Einstein's field equations in vacuum (Deng, Hu, Ding, Xue, \& Feng, 2009). Now we know the reason of that the law of Newton gravity does not satisfy Lorentz covariance is that Poisson equation of gravitational field is not Special Complete physical equation (Liu, 2014). The Requirement of Special Completeness (RSC) is that the physical equations used to describe the dynamics of matter and/or fields should include the descriptions that not only the matter and/or fields are at rest relative to an IRF, but also they move relative to this frame. The covariance is a basic characteristic of Special Complete physical equations. The main reason of that Einstein could not extend his SR without difficulty to establish the theory of spacetime of NRF was that he placed the propagation property of light in the central position of his theory because it is very difficult to synchronize the clocks in a NRF.

Now we are thinking whether we have an alternative option to establish a theory of the spacetime of NRF without gravitation? Maybe it is time to re-investigate the problems of that Einstein met at that time and to reconsider the opinions proposed by Einstein and hold by most physicists at present. Actually, the problem of NRF and the problem of gravitation are two separate problems and should not be considered together. In principle, the problem of gravitation in an IRF can be solved through finding the gravitational field equations of Lorentz covariance (Liu, 
2013), while the problem of spacetime of NRF can be solved independently. The accelerating motion is absolute and is relative to all IRF. Distinguishing an IRF and a NRF is a simple task. For instance, we can discover the rotation of the earth through observing the motion of a pendulum. When we are sitting in a vehicle, we can sense the acceleration of the vehicle. Moreover, in GR, it is also necessary to distinguish whether there is a gravitational field in the reference frame. This is merely another method of distinguishing an IRF and a NRF. Einstein's imaginary experiment of an accelerated cabin is somewhat self-entrapping. We have enough methods to ascertain a reference frame what kind of motive power (rocket or engine) make it move or how big mass this reference frame has. An IRF is a special case of the NRF and is also a more abstract reference frame. It can be predicted that an IRF is more superior at observing and describing the motion of matter. The reference frame at the Sun's center of mass is more superior comparing with the reference frame at the earth's center of mass. This can be seen by comparing the complexity of Ptolemy's geocentric model with Copernicus's heliocentric model. We will explain that the NRF can also be used to describe the motion of matter without introducing a gravitational field and employing Mach principle. Inertial force is not gravitation. Gravitational field is real field, while the inertial force is the manifestation of the deformation of spacetime due to the accelerating motion of the NRF.

In present paper, we will try to extend the Special Completeness to the General Completeness that has considered the structural characteristics of the spacetime of NRF and the principle of the equality of IRF to the principle of the equality of all reference frames, then, re-investigate the spacetime of NRF without considering gravitation. In Chapter II, we will investigate the characteristics and structure of spacetime of NRF; In Chapter III, we will discuss the objection of Mach principle; In Chapter IV, the requirement of general completeness and the principle of equality of all reference frames have been proposed; In Chapter V, the covariance of the physical equations of general completeness has been inferred.

\section{The Characteristics of the Spacetime in a NRF}

\subsection{Observations on a Rotating Plane}

Figure 1, in the inertial plane $\mathrm{K}^{\prime}$, there is a rotating plane $\mathrm{K}$ which rotates around the center $\mathrm{O}^{\prime}$ with the angular velocity $\omega$. The results observed on the inertial plane K' satisfy the pseudo-Euclidean geometry.

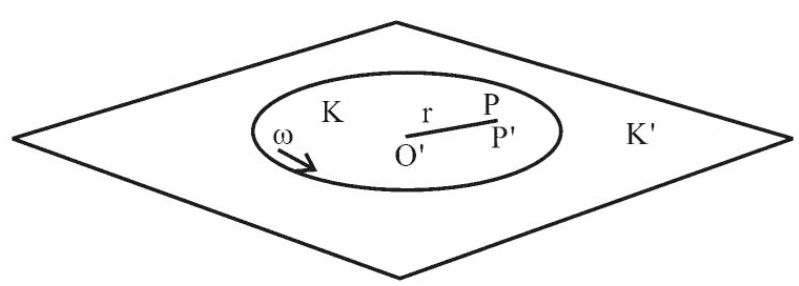

Figure 1. On the frames of reference $\mathrm{K}$ and $\mathrm{K}^{\prime}$, to describe an event

The observer on the plane $\mathrm{K}^{\prime}$ places a standard ruler and standard clock at point $\mathrm{P}^{\prime}$. The observer at the point $\mathrm{P}$ in the rotating plane $\mathrm{K}$ has an identical standard ruler and clock with point $\mathrm{P}^{\prime}$ and rotates with the rotating plane $\mathrm{K}$. During the infinitely short time interval when point $\mathrm{P}$ passes point $\mathrm{P}^{\prime}$, the infinitely small local space around $\mathrm{P}$ can be treated as an IRF. This is because every point in this local space has same velocity during this transient moment. In this local IRF $\mathrm{P}$, we observe the standard clock and ruler at point $\mathrm{P}^{\prime}$ on plane $\mathrm{K}^{\prime}$. We suppose that the clock at point $\mathrm{P}^{\prime}$ on plane $\mathrm{K}^{\prime}$ runs past $d \tau$ and the length of standard ruler at point $\mathrm{P}^{\prime}$ which is perpendicular to $\overline{O^{\prime} P^{\prime}}$ is $d l_{0}$. According SR, we obtain

$$
\begin{gathered}
d t=\frac{d \tau}{\sqrt{1-\frac{v_{p}^{2}}{c^{2}}}}, \\
d l=d l_{0} \sqrt{1-\frac{v_{p}^{2}}{c^{2}}} .
\end{gathered}
$$

Here $d t$ and $d l$ are respectively measured by the observer at point P. $c$ is the speed of light, $v_{p}$ is the speed of point $\mathrm{P}$ relative to plane $\mathrm{K}$ '.

According to formula (1) and (2), we can see that $d t$ and $d l$ are the function of spacetime. For the same $d \tau$ and $d l_{0}$ in the every point of the plane $\mathrm{K}$ ', the corresponding $d t$ and $d l$ in the every point of the plane $\mathrm{K}$ are 
not same because $v_{p}$ is a function of spacetime. Please notice here: it is different with previous people who standed on the IRF to observe the rulers and the clocks on the NRF (the rotating plane), whereas we stand on the NRF to measure the rulers and the clocks on the IRF. We stand on a NRF by observing the motion of matter or fields (or rulers and clocks) to determine the characteristics of the spacetime of the NRF.

\subsection{The Structure of Spacetime in NRF}

The observation of spacetime on the rotating plane indicates that any point of the spacetime in a NRF is a local IRF. The differences observed between the local IRF and the corresponding point of the spacetime in the IRF come down to the differences of spacetime. The moving status of a NRF can be ascertained, as soon as an IRF is selected, thereby, the differences of spacetime are certain between every local IRF in NRF and the corresponding point of spacetime in the IRF. The NRF is the united body of infinite local IRF. In each local IRF, the results observed are different for the same ruler and clock at the corresponding point of spacetime in the IRF. Therefore, the spacetime of a NRF is inhomogeneous.

We cannot establish a homogeneous solid Cartesian coordinate system (note: the coordinate system and the reference frame are two different concepts) in a whole NRF, and just can introduce the curvilinear coordinate system. The coordinate transformation can only be nonlinear transformations between the NRF and the IRF. We suppose that there is a NRF Q and an IRF Q', the coordinate system in the reference frame $\mathrm{Q}$ is $x^{\mu}$, the coordinate system in the reference frame $\mathrm{Q}^{\prime}$ is $x^{i}$. The coordinate transformation of the two spacetimes is

$$
x^{\prime i}=x^{\prime i}\left(x^{\mu}\right)
$$

Here, $i$ and $\mu$ take $0,1,2,3$. In the reference frame Q', the spacetime is Minkowski spacetime and the metric of spacetime is

$$
d s^{2}=\eta_{i j} d x^{\prime i} d x^{\prime j}
$$

here $\eta_{i j}$ is the metric tensor of Minkowski spacetime.

According to the formula (3), we obtain

$$
\begin{aligned}
& d x^{\prime i}=\frac{\partial x^{\prime i}}{\partial x^{\mu}} d x^{\mu}, \\
& d x^{j}=\frac{\partial x^{\prime j}}{\partial x^{\nu}} d x^{\nu} .
\end{aligned}
$$

Substituting above two formulas to (4), we gain

Introduce

$$
d s^{2}=\eta_{i j} \frac{\partial x^{\prime i}}{\partial x^{\mu}} \frac{\partial x^{\prime j}}{\partial x^{v}} d x^{\mu} d x^{v}
$$

$$
\gamma_{\mu v}=\eta_{i j} \frac{\partial x^{\prime i}}{\partial x^{\mu}} \frac{\partial x^{\prime j}}{\partial x^{v}}
$$

$\gamma_{\mu v}$ is obviously the function of $x^{\mu}$. We have

$$
d s^{2}=\gamma_{\mu \nu} d x^{\mu} d x^{v}
$$

In any certain neighborhood of a point of spacetime, $\gamma_{\mu \nu}$ is approximately a constant, so this neighborhood can also approximately be seen as a local pseudo-Euclidean spacetime. This is what we call the local IRF. Now we can conclude that the structure of spacetime in a NRF Q is a Riemann spacetime, its metric is formula (9), and the local pseudo-Euclidean spacetime in any point of spacetime is the tangent space of the Riemann spacetime.

We are aware that, in a NRF, the Riemann-Christoffel symbol cannot be zero, because we cannot establish a Cartesian coordinate system in a NRF to describe the motion of matter. This is the intrinsic characteristic of inhomogeneous and deformed spacetime in a NRF. It is worthy to clarify that the characteristics of spacetime are decided by the motion of matter relative to the reference frame, and the moving status of the reference frame will naturally affect the observation and description of the motion of matter, thereby, will decide its structure of spacetime. It is not related to gravitation! We cannot find a coordinate transformation in a NRF to eliminate the 
inhomogeneous and deformation of spacetime. However we can find a coordinate transformation, for instance, one may use formula (3), to eliminate its inhomogeneous and deformation, but actually this transformation has already transferred from a NRF to an IRF. Note: some coordinate transformations are in one reference frame, these kinds of coordinate transformations do not change its structure of spacetime; while some transformations, for instant, transforming from a NRF to an IRF, can change the structure of spacetime. The structure of spacetime of a NRF belongs to Riemann manifold, but its Riemann curvature is zero. It is also different with the structure of spacetime of the IRF. We call these kinds of spacetime as Non-inertia Spacetime, and call the flat, isotropic and homogeneous spacetime as Inertia Spacetime.

If one asks why a free body in an IRF is always at rest or maintains rectilinear motion with a uniform velocity while a free body in a NRF move along with a curved line, the answer is because the spacetime in the IRF is isotropic and homogeneous, while the spacetime in the NRF is inhomogeneous and deformed. In Physics, the IRF is a special situation of the NRF whose acceleration is zero. In Mathematics, Minkowski spacetime of the IRF (or Inertia Spacetime) is a special situation of Non-inertia Spacetime of the NRF when the metric tensor $\gamma_{\mu \nu}=\eta_{i j}$. Both are the special situation of Riemannian manifold.

\section{The Objection of Mach's Principle}

The idea that inertia represents the effects of interactions with faraway matter was first developed by Ernst Mach in the nineteenth century (Feynman, 1995, pp.70). This idea called Mach Principle. No matter if this was Mach's real intention (Barbour \& Pfister, 1995), anyway, it was one of the powerful ideas that Einstein had in mind as he constructed his theory of gravitation (Feynman, 1995, pp.70). Einstein wrote to Ernst Mach to express his appreciation for the inspiration that he had derived for his endeavors from Mach's ideas (Misner, Thorne, \& Wheeler, 1973, p. 543).

In a NRF, all free particles move with an acceleration even if there are no external forces acted on. The reason for this accelerating motion, according to Mach's principle, is that the free particle is acted on by the gravitation produced by the accelerating motion of the matter in universe relative to the reference frame. If we consider an empty space containing only an accelerated reference frame and a test particle, the inertial effect will occur. The motion of the test particle would certainly not be affected by faraway galaxies which do not exist. Until now, no experiment supports Mach principle (Hughes, Robinson, \& Beltran-Lopez, 1960; Ivash, \& Sweet, 1961; Liu, 2004, p. 96). To say that there is a gravitational field is also false, because the source of the gravitational field does not exist! The gravitational field like the electromagnetic field and other fields is a real matter field which is related to the gravitational source, the energy-momentum tensor of matter (Liu, 2013). If there is no matter, where does the gravitational field come from? The motion of the test particle is only related to the accelerated reference frame. Furthermore, the accelerating motion of the test particle relative to the NRF occurs instantaneously and the speed of propagation of gravitational force cannot be infinite.

We think that the motion of a free particle with respect to a NRF can only be attributed to the deformation of spacetime. This is the same as how the differences of physical observation between the IRFs are attributed to the differences of the spacetime of the IRFs (Liu, 2014). In the Chapter II, we have already discussed that the spacetime of a NRF is different from the spacetime of an IRF. The spacetime of a NRF is inhomogeneous or deformed. Certainly, the root cause of the inhomogeneity or deformation is still the accelerating motion of the reference frame. The accelerating motion of the reference frame is relative to the any IRF. Newton's absolute space cannot be found. A convenient choice of the IRF is the one which is at rest relative to a faraway galaxy. At least, this is relatively better one. This probably is Mach's original idea (Barbour \& Pfister, 1995). In his book, The Science of Mechanics, Mach [(1912), Chapter 2, section 6] had reasoned that it could not make sense to speak of the acceleration of a mass relative to absolute space. Anyone trying to clear physics of mystical ideas would do better to speak of acceleration relative to the distant stars (Misner, Thorne, \&Wheeler, 1973, pp.543).

Einstein's opinion with regard to the inertial force is the precondition that he proposed the principle of general relativity. It seems that only the inertial force is regarded as gravitation, then, IRF and NRF can be regarded as equality for describing the physical phenomena. Einstein's logical clue is that a NRF is regarded as an IRF with a gravitational field, then, according to the principle of special relativity, the both frames are equality. This logic is incorrect! It makes the theory of the spacetime of NRF complicated. We will see in following chapter that all reference frames are equal to describe the motion of matter without considering gravitation, only considering the structure of spacetime of reference frames. 


\section{Basic Principles}

\subsection{The Reqirement of General Completeness}

A new requirement to the physical equations has been proposed (Liu, G. 2014 ), namely, the physical equations used to describe the dynamics of matter and/or fields should include the descriptions that not only the matter and/or fields are at rest relative to an IRF, but also they move relative to this reference frame. Combining this requirement and the equality of inertial reference frames, a new approach to the theory of Lorentz covariance without light has been proposed. The covariance of physical equations is attributed to the completeness of physical equations and the equality of inertial reference frames. Actually, according to above analysis we realize that, regardless of the IRF or the NRF, all of them can be used to observe and describe the motion of matter, their differences are the differences of the characteristics of their spacetime. To be exact, its geometrical characteristics of the spacetime are different, while the Minkowski spacetime is actually the special situation of Non-inertia Spacetime. Certainly the differences of the spacetime are caused by the motion of matter relative to the reference frame. The reference frame moved in different status has different structure of spacetime. So called inertial force represents the effects of the deformation of spacetime. Therefore, if we extend the Special Completeness to the NRF, a more general condition to replace the "the IRF" is that "the structural characteristic of the spacetime of reference frame has been considered in the physical equations". Actually, in the statement of the Special Completeness, the condition "the IRF" just requires us to consider the structural characteristic of Minkowski spacetime in the equations. Therefore, we state the Requirement of General Completeness like this: the physical equations used to describe the dynamics of matter and/or fields should include the descriptions that not only the matter and/or fields are at rest, but also they move (uniformly rectilinearly or acceleratedly) relative to this reference frame, and the structure of the spacetime of reference frame has been considered. We have known that tensor expression of physical equations is best way to manifest the Requirement of the General Completeness. Here, the tensor expression has included the considerations of the metric tensor of spacetime. Therefore, the Requirement of General Completeness (RGC) can also be stated like this: the physical equations used to describe the dynamics of matter and/or fields should be the tensor equations.

\subsection{The Principle of Equality of All Reference Frames}

What is the relationship between the physical equations of general completeness in each reference frame? For one series of motions of matter, all of the observers in every reference frame can establish their physical equations of general completeness. We have already known that, in the situation which metric tenser $\gamma_{\mu v}$ of spacetime can be expressed as Minkowski metric $\eta_{i j}$, namely when the reference frame is an IRF, the form of the physical equations of general completeness (now we should call them the physical equations of special completeness) in every reference frame is same. In this situation, because the metric tensor of the spacetime in every reference frame is same, the physical equations of general completeness are not only identical in form, but also completely identical. For non-inertial reference frames, the metric tensors $\gamma_{\mu \nu}$ in every reference frame are different, and that the metric tensor will affect its meaning of the physical equations of general completeness. This is because the difference of structure of spacetime reflects in the difference of metric tensor. Therefore, any reference frame can be used to describe the motion of matter and/or field and the physical equations or laws of general completeness in all reference frames are identical. We call this statement as the Principle of Equality of All Reference Frames (PERF).

\section{Covariance of the Physical Equations of General Completeness}

Supposing there are a series of motions of same kind of matter, for instance, the electromagnetic motions, for two arbitrarily selected reference frames, by making a series of observations, the observers in the reference frames can establish their own physical equations of general completeness. According to the PERF, we know that the equations of general completeness are identical. Due to the difference of moving status of two reference frames, their observations regarding the details of moving matter (for instance, the electromagnetic field of a moving charger Q) are different. Just like that has been pointed out by G. Liu (Liu, 2014): the differences of observation finally only attribute to the differences of spacetime. Here obviously indicate again that, spacetime is the extensibility and the continuity of motion of matte relative to a reference frame; if there is no reference frame, we could not describe the motion of matter; if there is no motion of matter, space and time will be meaningless. The differences of spacetime embody in the transformations of spacetime coordinates between the reference frames. Therefore, according to the PERF, we deduce that the equations of general completeness remain identical under coordinate transformations between all reference frames. We call this statement as the Covariance of the Equations of General Completeness. 
How to establish the physical equations of general completeness? The covariant differential has two characteristics: first, it changes from tensor to tensor; second, when the reference frame is IRF and we select the Cartesian coordinates, therefore, $\Gamma_{v \lambda}^{\mu}=0$, it becomes to normal differential. These characteristics enlighten us by using the following arithmetical method to establish the equations of general completeness: write the equations of special completeness in terms of tensor forms in IRF, then, replace $\eta_{i j}$ by using $\gamma_{\mu v}$, and replace all of the differentials by using the covariant differentials. The equations obtained are covariant with respect to all transformations of spacetime coordinates between all reference frames, and are Special Complete with respect to the inertial reference frames.

Both of changing the physical equations into the general complete form and changing the physical equations into the general relativistic form are similar. We can borrow this kind of achievements of General Relativity, but it must be stressed that the equations of general completeness are not related to the principle of equivalence and gravitational field.

\section{Summary}

The characteristics of spacetime of NRF have been discussed without considering gravitation by proposing the RGC and the PERF. The spacetime of NRF is inhomogeneous or deformed. The inertial force is only attributed to the deformation of spacetime. The IRF and the NRF can be distinguished and the differences between them are the structures of spacetime. The accelerating motion is absolute. The superior reference frame on the observation and description does exist. That is the IRF. All physical equations should be written as the general complete tensor equations, thereby, they are covariant with respect to the coordinate transformations between arbitrary reference frames. The Minkowski spacetime in the IRF is its special case. A real gravitational field can been described in IRF and NRF (Liu, 2013).

\section{Acknowledgments}

I am grateful to all my family members, colleagues and friends for their encouraging and support.

\section{References}

Barbour, J. B., \& Pfister, H. (1995). Mach's Principle: From Newton's Bucket to Quantum Gravity. Birkhauser, Boston.

Deng, J. X. Hu, Ding, Y., Xue, D., \& Feng, Y. (2009). On General Møller Transformation, Commun. Theor. Phys., 52, 75. (Beijing, China). https://doi.org/10.1088/0253-6102/52/1/16

Einstein, A. (1920). Relativity — the special \& general Theory (3rd Ed.). METHUEN \& GO. LTD., LONDON.

Einstein, A. (1970). The Meaning of Relativity (5th Ed.). Princeton University Press, Princeton, New Jersey.

Feynman, R. (1995). Feynman Lecture on Gravitation. Addison-Wesley Publishing Company, New York.

Hughes, V. W., Robinson, H. G., \& Beltran-Lopez, V. (1960). Upper Limit for the Anisotropy of Inertial Mass from Nuclear Resonance Experiments. Phys. Rev. Lett, 4, 342. https://doi.org/10.1103/PhysRevLett.4.342

Ivash, E. V., \& Sweet, R. F. (1961). Mass Anisotropy and Planetary Motion. IL Nuovo Cimento, XXII(2), 352. https://doi.org/10.1007/BF02783025

Liu, G. (2013). Remannian Spacetime, de Donlder Conditions and Gravitational Field in Flat Spacetime, International Journal of Astronomy and Astrophysics, 3(1), 8-9. https://doi.org/10.4236/ijaa.2013.31002

Liu, G. (2014 ). A New Approach to Special Relativity. Physics Essays, 27(1). https://doi.org/10.4006/0836-139827.1.173

Liu, L. (2004). General Relativity (p. 8). Advanced Education Publishing Company, Beijing.

Misner, C. W., Thorne, K. S., \& Wheeler, J. A. (1973). Gravitation, W. H. Freeman and Company, San Francisco. Moller, C. (1952). The Theory of Relativity. Clarendon, Oxford, Chap. VIII.

Weinberg, S. (1972). Gravitation and Cosmology: Principles and Applications of the General Theory of Relativity. John Wiley \& Sons, Inc., New York.

\section{Copyrights}

Copyright for this article is retained by the author(s), with first publication rights granted to the journal.

This is an open-access article distributed under the terms and conditions of the Creative Commons Attribution license (http://creativecommons.org/licenses/by/4.0/). 